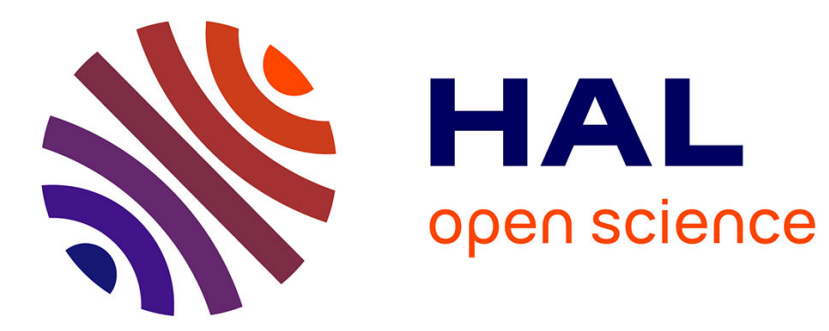

\title{
New empirical relations for stopping power and range of charged particles
}

\author{
A.K. Chaubey, H.V. Gupta
}

\section{To cite this version:}

A.K. Chaubey, H.V. Gupta. New empirical relations for stopping power and range of charged particles. Revue de Physique Appliquée, 1977, 12 (2), pp.321-329. 10.1051/rphysap:01977001202032100 . jpa00244166

\section{HAL Id: jpa-00244166 https://hal.science/jpa-00244166}

Submitted on 1 Jan 1977

HAL is a multi-disciplinary open access archive for the deposit and dissemination of scientific research documents, whether they are published or not. The documents may come from teaching and research institutions in France or abroad, or from public or private research centers.
L'archive ouverte pluridisciplinaire HAL, est destinée au dépôt et à la diffusion de documents scientifiques de niveau recherche, publiés ou non, émanant des établissements d'enseignement et de recherche français ou étrangers, des laboratoires publics ou privés. 


\title{
NEW EMPIRICAL RELATIONS FOR STOPPING POWER AND RANGE OF CHARGED PARTICLES
}

\author{
A. K. CHAUBEY and H. V. GUPTA (*)
}

Department of Physics, Aligarh Muslim University, Aligarh 202001, India

\begin{abstract}
Résumé. - Nous avons établi quelques relations empiriques donnant le pouvoir d'arrêt des protons et des particules $\alpha$ dans un domaine d'énergie allant de 0,7 à $12 \mathrm{MeV} / \mathrm{uma}$. Les relations parcours-énergie ont été obtenues par intégration directe des formules du pouvoir d'arrêt. Ces relations ont été appliquées à la détermination du parcours et du pouvoir d'arrêt dans les détecteurs à semiconducteurs. Certaines autres applications de ces formules seront également discutées.
\end{abstract}

Abstract. - We have introduced some new empirical relations for stopping powers of protons and alpha particles in the energy range 0.7 to $12 \mathrm{MeV} / \mathrm{amu}$. The range formulas were obtained by directly integrating the stopping power formulas of protons and alpha particles. These relations have been also used to find out stopping powers and ranges in semiconductor detectors. Practical applications of the empirical relations are also discussed.

1. Introduction. - A precise knowledge of the stopping power and range - energy relationship for heavy charged particles in various media is of considerable interest both from the practical as well as theoretical point of view. Many experimental as well as theoretical studies have been made with the object of establishing standard range energy relations. The subject has been reviewed in last two decades by several authors such as Taylor [1], Bethe and Askin [2], Allision and Warshaw [3], Uehling [4] and by Barkas and Berger [5]. Most of the experimental data has been compiled by Whaling [6] and Bichsel [7] in the form of tables. There have been several discussions and compilations on the energy - loss and range of heavy charged particles. Most of the work [8-28] either depends on the use of fairly complicated semiempirical formulas derived from the Bethe-Bloch expression of stopping power or on entirely empirical formulas extracted from the experimental information. The old empirical formulas are in great error due to lack of correct experimental information at that time. Moreover some relations are valid only for specific values of $Z$ and in a small energy region. In this paper, we also present empirical formulas for stopping power and range of charged particles.

2. Empirical relation for stopping power. - We have arrived at the following empirical relation for the stopping power of protons ;

$$
-\frac{\mathrm{d} E}{\rho \mathrm{d} X}=\frac{a}{A} E^{-b} Z^{c \log E+d} .
$$

(*) Present address : Meteorological Office, Govt. of India, Bombay Airport, Bombay, India.
The appropriate values of the constants $a, b, c$, and $d$ are $a=915.0, b=0.85, c=0.145, d=0.635$.

Here $\rho, A$ and $Z$ denote the density, atomic weight and atomic number of the stopping material while $E$ is the kinetic energy of the particle in MeV/amu. The eq. (1) is found to be valid in the energy region 0.7 to $12 \mathrm{MeV} / \mathrm{amu}$. The stopping power is in $\mathrm{MeV} \mathrm{cm} / \mathrm{gm}$. The constants $c$ and $d$ are found to be independent of particle type and were obtained by fitting Northcliffe and Schilling [28] (hereafter referred to as NS) stopping power values by the least squares method while the constants $a$ and $b$ were extracted using the experimental data of Whaling [6] and Anderson et al. [29] and also NS data towards lower energies.

The stopping power for the ions heavier than protons can be found by the expressions given by Pierce and Blann [25]

$$
\left(-\frac{\mathrm{d} E}{\rho \mathrm{d} X}\right)_{\mathrm{H}}=\frac{z_{\text {eff }}^{2}}{\gamma_{\mathrm{p}}^{2}}\left(-\frac{\mathrm{d} E}{\rho \mathrm{d} X}\right)_{\mathrm{p}}
$$

where

$$
z_{\text {eff }}^{2}=\gamma^{2} z^{2}
$$

Here we are concerned with the energy $0.7 \mathrm{MeV}$ and above, therefore, for protons we have taken $\gamma_{p}=1$. Substituting eq. (1) in eq. (2) we get,

$$
\left(-\frac{\mathrm{d} E}{\rho \mathrm{d} X}\right)_{\mathrm{H}}=\gamma^{2} z^{2} a E^{-b} Z^{c \log E+d} \text {. }
$$

In eq. (3) the fractional effective charge $\gamma$ of the ion of energy $E(\mathrm{MeV} / \mathrm{amu})$ can be estimated from Booth and Grant's [30] empirical formula. The formula is given by $\gamma^{2}=f\left(E z^{-4 / 3}\right)$; where,

$$
f(x)=1-\exp \left(-24.73 x+247.6 x^{2}-1131 x^{3}\right) \text {. }
$$


For the calculation of the stopping power of alpha particles from eq. (3), we assume that above $4 \mathrm{MeV}$ the fractional effective charge of the alpha particles is $\gamma^{2}=1$. Below $4 \mathrm{MeV}$ i.e. from 0.7 to $1.0 \mathrm{MeV} / \mathrm{amu}$, we have fitted the NS data for alpha particles by the least squares method keeping $c$ and $d$ constant and got $a=3574, b=0.84$, therefore between 0.7 to $1.0 \mathrm{MeV} /$ amu for alpha particles, eq. (1) is modified as

$$
\left(-\frac{\mathrm{d} E}{\rho \mathrm{d} X}\right)=\frac{3574}{A} E^{-0.84} Z^{0.145 \log E+0.635}
$$

Above $1 \mathrm{MeV} / \mathrm{amu}$ we can use eq. (3) by simply putting $z=2$ and $\gamma=1$.

3. Range-Energy relations. - The range of a charged particle is computed by numerical integration of the stopping power. The range $R$ in the continuous slowing down approximation (csda) is given as

$$
R=\int_{E_{\min }}^{E_{\max }}\left(-\frac{\mathrm{d} E}{\rho \mathrm{d} X}\right)^{-1} \mathrm{~d} E+R\left(E_{\min }\right)
$$

where $R\left(E_{\min }\right)$ is the measured range at energy $E_{\text {min }}$ which is added to the integral equation (6) and treated as a constant for a particular particle and material.

For the calculation of ranges for protons we have taken $E_{\min }$ as $1 \mathrm{MeV}$ as much data is available at $1 \mathrm{MeV}$. Substituting eq. (1) into eq. (6) and converting energy units from $\mathrm{MeV}$ to $\mathrm{MeV} / \mathrm{amu}$ we get,

$R_{\mathrm{p}}=\int_{E_{1}}^{E} m_{\mathrm{p}}\left[\frac{a}{A} E^{-b} Z^{c \log E+d}\right]^{-1} \mathrm{~d} E+R_{1}\left(E_{1}\right)$.

Here $m_{\mathrm{p}}$ is the mass of the proton.

After integration and putting in the values of the constants we get,

$$
\begin{aligned}
R_{\mathrm{p}}= & m_{\mathrm{p}}\left[\frac{A}{915 \times 1.85 Z^{0.635}\left(1-\frac{0.145 \log Z}{1.85}\right)}\right] \times \\
& \times\left[E^{1.85} Z^{-0.145 \log E}-E_{1}^{1.85} Z^{-0.145 \log E_{1}}\right]+R_{1}\left(E_{1}\right) .
\end{aligned}
$$

The $R_{1}\left(E_{1}\right)$ is the experimental range of the proton at energy $E_{1}$ which slightly differs from the calculated range at the same energy $E_{1}$. Therefore, the second term in the second bracket of eq. (8) can be combined with $R_{1}\left(E_{1}\right)$ and we may define a correction term $F_{\mathrm{p}}$ to the range in a specific medium as,

$$
F_{\mathrm{p}}=R_{1}\left(E_{1}\right)-m_{\mathrm{p}} G_{\mathrm{p}} E_{1}^{1.85} Z^{-0.145 \log E_{1}}
$$

where

$$
G_{\mathrm{p}}=\left[\frac{A}{915 \times 1.85 Z^{0.635}\left(1-\frac{0.145 \log Z}{1.85}\right)}\right] .
$$

Therefore, eq. (8) reduces to

$$
R_{\mathrm{p}}=m_{\mathrm{p}} G_{\mathrm{p}} E^{1.85} Z^{-0.145 \log E}+F_{\mathrm{p}} .
$$

This equation gives the ranges of protons in $\mathrm{gm} / \mathrm{cm}^{2}$ in solid medium in the energy region 0.7 to $12.0 \mathrm{MeV} / \mathrm{amu}$.

The range of other heavier particles of energy $E_{\mathrm{i}}(\mathrm{MeV})$ graeter than $\beta_{\mathrm{i}}$ where $\beta_{\mathrm{i}}=0.04 z_{\mathrm{i}}^{2 / 3}$ can be calculated from the proton ranges; i.e.

$$
\begin{aligned}
R_{\mathrm{i}}\left(E_{\mathrm{i}}, z_{\mathrm{i}}, m_{\mathrm{i}}\right) & =R_{\mathrm{i}}\left(E_{\mathrm{ci}}, z_{\mathrm{i}}, m_{\mathrm{i}}\right)+\frac{m_{\mathrm{i}} / m_{\mathrm{p}}}{z_{\mathrm{i}}^{2}} \times \\
& \times\left[R_{\mathrm{p}}\left(E_{\mathrm{i}} m_{\mathrm{p}} / m_{\mathrm{i}}\right)-R_{\mathrm{p}}\left(E_{\mathrm{ci}} m_{\mathrm{p}} / m_{\mathrm{i}}\right)\right]
\end{aligned}
$$

where the subscript $\mathrm{i}$ refers to ion and $\mathrm{p}$ refers to proton. $E_{\mathrm{ci}}$ is the energy corresponding to $\beta_{\mathbf{i}}=0.04 z_{\mathrm{i}}^{2 / 3}$. Again the problem arises for getting $R_{\mathrm{i}}\left(E_{\mathrm{ci}}, z_{\mathrm{i}}, m_{\mathrm{i}}\right)$, i.e. the range of the ion below $E_{\mathrm{i}} \leqslant E_{\mathrm{ci}}$. This can either be computed by numerical integration of the theoretical expression in conjunction with shell corrections or be measured experimentally.

The range of alpha particle between $0.7 \mathrm{MeV}$ to $1 \mathrm{MeV} / \mathrm{amu}$ can be calculated from integration of eq. (5) similarly as in the case of protons provided that the stopping power of alpha particles is known below $0.7 \mathrm{MeV} / \mathrm{amu}$. We obtain

$$
\begin{aligned}
R_{\alpha}= & m_{\alpha}\left[\frac{A}{1.84 \times 3574 \times Z^{0.635}\left(1-\frac{0.145 \log Z}{1.84}\right)}\right] \times \\
& \times\left[E^{1.84} Z^{-0.145 \log E}-E_{2}^{1.84} Z^{-0.145 \log E_{2}}\right]+R_{2}\left(E_{2}\right),
\end{aligned}
$$

or

$$
R_{\alpha}=m_{\alpha} G_{\alpha} E^{1.84} Z^{-0.145 \log E}+F_{\alpha},
$$

where

$$
F_{\alpha}=R_{2}\left(E_{2}\right)-m_{\alpha} G_{\alpha} E_{2}^{1.84} Z^{-0.145 \log E_{2}} .
$$

Here $G_{\alpha}$ is equal to the factor inside the first bracket of eq. (13) and $F_{\alpha}$ is the correction term obtained from eq. (13). $R_{2}\left(E_{2}\right)$ must be the known range of alpha particles at $0.7 \mathrm{MeV} / \mathrm{amu}$ (or $0.75 \mathrm{MeV} / \mathrm{amu}$ ). At the cost of a little accuracy eq. (14) may be used directly for calculating particle ranges between $0.7 \mathrm{MeV} / \mathrm{amu}$ to $12 \mathrm{MeV} / \mathrm{amu}$, otherwise above $4 \mathrm{MeV}$ the ranges can be obtained using eq. (11) and (12). Now putting eq. (11) into eq. (12) we get,

$R_{\alpha}=\frac{G_{\mathrm{p}} m_{\alpha}}{z^{2}} \times$

$$
\times\left[E^{1.85} Z^{-0.145 \log E}-E_{2}^{1.85} Z^{-0.145 \log E_{2}}\right]+R_{2}\left(E_{2}\right) ;
$$

letting

$$
F_{\alpha \alpha}=R_{2}\left(E_{2}\right)-\frac{G_{\mathrm{p}} m_{\alpha}}{z^{2}} E_{2}^{1.85} Z^{-0.145 \log E_{2}},
$$

we get

$$
R_{\alpha}=\frac{G_{\mathrm{p}} m_{\alpha}}{z^{2}} E^{1.85} Z^{-0.145 \log E}+F_{\alpha \alpha}
$$


Therefore, if we know $R_{2}\left(E_{2}\right)$ at $4 \mathrm{MeV}$ eq. (17) can be used to obtain ranges of alpha particles up to $48 \mathrm{MeV}$. Thus ranges from 3 to $48 \mathrm{MeV}$ can be calculated for alpha particles from our relations. (In fact one can stretch the empirical formula from 2.5 to $50 \mathrm{MeV}$ without much error.)

4. Stopping power and range for compound targets. The stopping power of a compound medium can be obtained by the additivity theorem

$$
\left(-\frac{\mathrm{d} E}{\rho \mathrm{d} X}\right)_{\text {compound }}=\frac{1}{M} \sum_{i} N_{i} A_{i}\left(-\frac{\mathrm{d} E}{\rho \mathrm{d} X}\right)_{i} .
$$

We have made use of eq. (18) to calculate the energy loss in CdTe, GaAs and AgBr. Here $M$ is the molecular weight of the compound medium containing $\mathrm{N}_{i}$ atoms of atomic weight $A_{i}$. For example for CdTe the stopping power can be given as

$$
\begin{aligned}
& \left(-\frac{\mathrm{d} E}{\rho \mathrm{d} X}\right)_{\mathrm{CdTe}}=\frac{1}{240.02} \times \\
& \quad \times\left[112.41\left(-\frac{\mathrm{d} E}{\rho \mathrm{d} X}\right)_{\mathrm{Cd}}+127.61\left(-\frac{\mathrm{d} E}{\rho \mathrm{d} X}\right)_{\mathrm{Te}}\right]
\end{aligned}
$$

Barkas [31], while studying energy losses in nuclear research emulsions, gave the following formula for the range in $n$-component materials

$$
\frac{1}{R}=\sum_{i=1}^{n} \frac{f_{i}}{R_{i}}
$$

where $R_{i}$ is the calculated range in the $i$-th component and $f_{i}$ is the fraction by weight of that constituent of the stopping medium. This formula has also been used by Barkas and Berger [5] while presenting the table for stopping power of protons in several compound materials. Eq. (20) has been found to be very accurate although not rigorously derivable. Eq. (20) can be written for CdTe as

$$
R(\mathrm{CdTe})=\frac{R(\mathrm{Cd}) R(\mathrm{Te})}{0.4684 R(\mathrm{Te})+0.5316 R(\mathrm{Cd})} .
$$

5. Results. - Using the empirical relations given above, we have calculated stopping powers and ranges from 0.7 to $12 \mathrm{MeV} / \mathrm{amu}$ for $\mathrm{Al}, \mathrm{Cu}, \mathrm{Ge}, \mathrm{Zr}, \mathrm{Ag}, \mathrm{Eu}$, $\mathrm{Au}$ and for compounds $\mathrm{CdTe}$ and GaAs. In these calculations values of starting ranges were taken from the literature and these are tabulated in table I with their references. Figures 1 to 5 displav the stopping powers versus energy and figures 6 to 10 show the ranges versus energy in $\mathrm{Al}, \mathrm{Cu}, \mathrm{Ge}, \mathrm{Ag}, \mathrm{Au}, \mathrm{GaAs}$

\begin{tabular}{|c|c|}
\hline Substance & $R_{1}\left(E_{1}\right) ; \underset{\left(\mathrm{mg} / \mathrm{cm}^{2}\right)}{\text { Range of } 1 \mathrm{MeV}}$ protor \\
\hline $\mathrm{Al}$ & 3.967 (Ref. [28]) \\
\hline $\mathrm{Cu}$ & 6.12 (Ref. [6]) \\
\hline $\mathrm{Ga}$ & 6.384 (Ref. [5]) Interpolation \\
\hline $\mathrm{Ge}$ & 6.533 (Ref. [28]) \\
\hline As & 6.643 (Ref. [5]) Interpolation \\
\hline $\mathrm{Ag}$ & 7.697 (Ref. [28]) \\
\hline $\mathrm{Cd}$ & 8.326 (Ref. [5]) Interpolation \\
\hline $\mathrm{Te}$ & 9.103 (Ref. [5]) Interpolation \\
\hline $\mathrm{Au}$ & 12.602 (Ref. $[28])$ \\
\hline
\end{tabular}

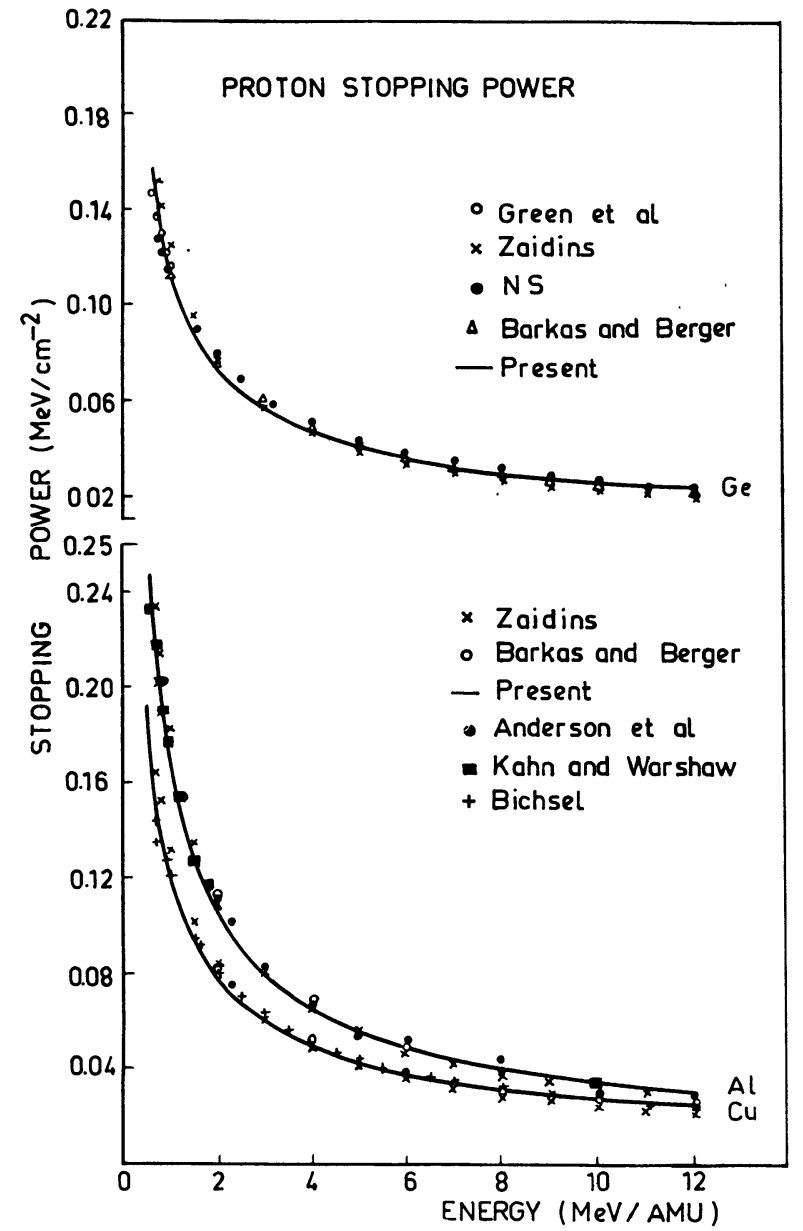

Fig. 1. - Empirical values for stopping power of protons versus energy in $\mathrm{Al}, \mathrm{Cu}$ and $\mathrm{Ge}$ along with other authors results.

TABLE I
$R_{2}\left(E_{2}\right)$; Range of alpha particles $\left(\mathrm{mg} / \mathrm{cm}^{2}\right)$
$0.7 \mathrm{MeV} / \mathrm{amu}=2.745$ (Ref. [28])
$0.75 \mathrm{MeV} / \mathrm{amu}=5.10$ (Ref. [6])
$0.70 \mathrm{MeV} / \mathrm{amu}=4.70$ (Ref. [28]) Interpolation
$0.70 \mathrm{MeV} / \mathrm{amu}=4.782$ (Ref. [28])
$0.70 \mathrm{MeV} / \mathrm{amu}=4.90$ (Ref. [28]) Interpolation
$0.70 \mathrm{MeV} / \mathrm{amu}=5.622$ (Ref. [28])
$0.70 \mathrm{MeV} / \mathrm{amu}=6.42$ (Ref. [28]) Interpolation
$0.70 \mathrm{MeV} / \mathrm{amu}=6.83$ (Ref. [28]) Interpolation
$0.70 \mathrm{MeV} / \mathrm{amu}=9.687$ (Ref. [28]) 


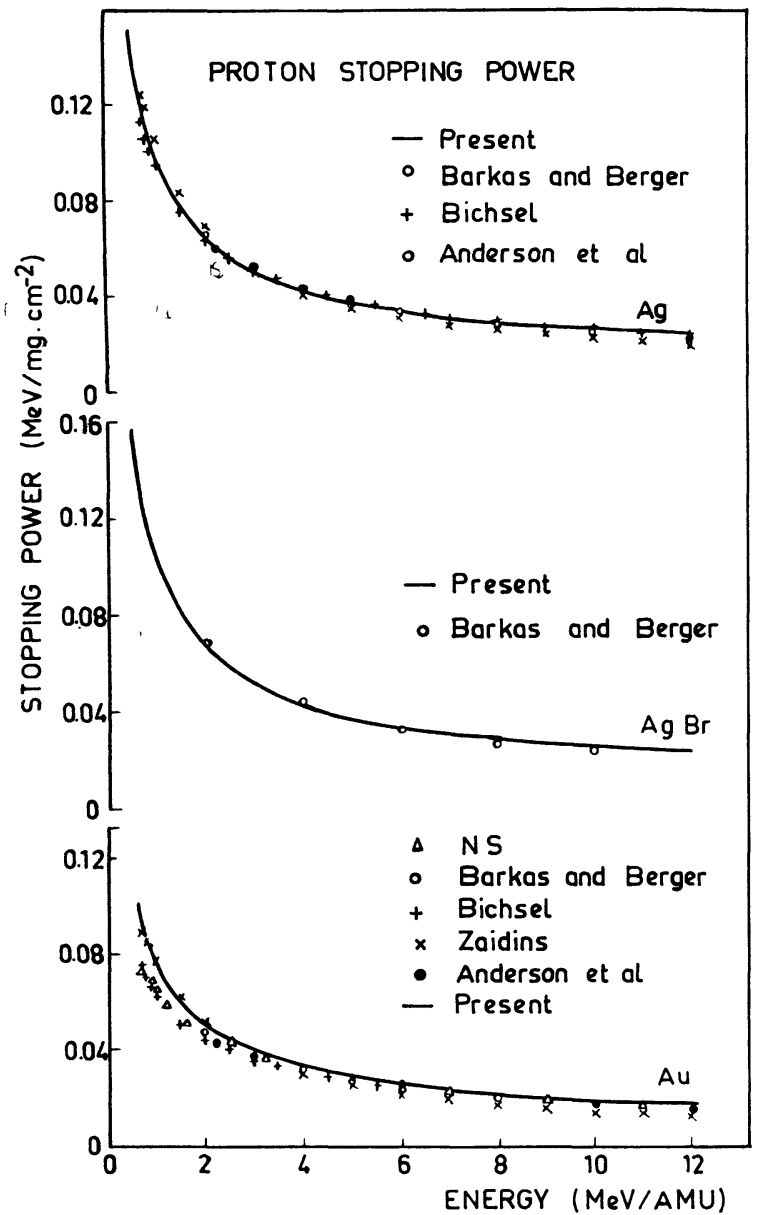

FIG. 2. - Empirical values for stopping power of protons versus energy in $\mathbf{A g}, \mathrm{AgBr}$ and $\mathrm{Au}$ along with other workers results.

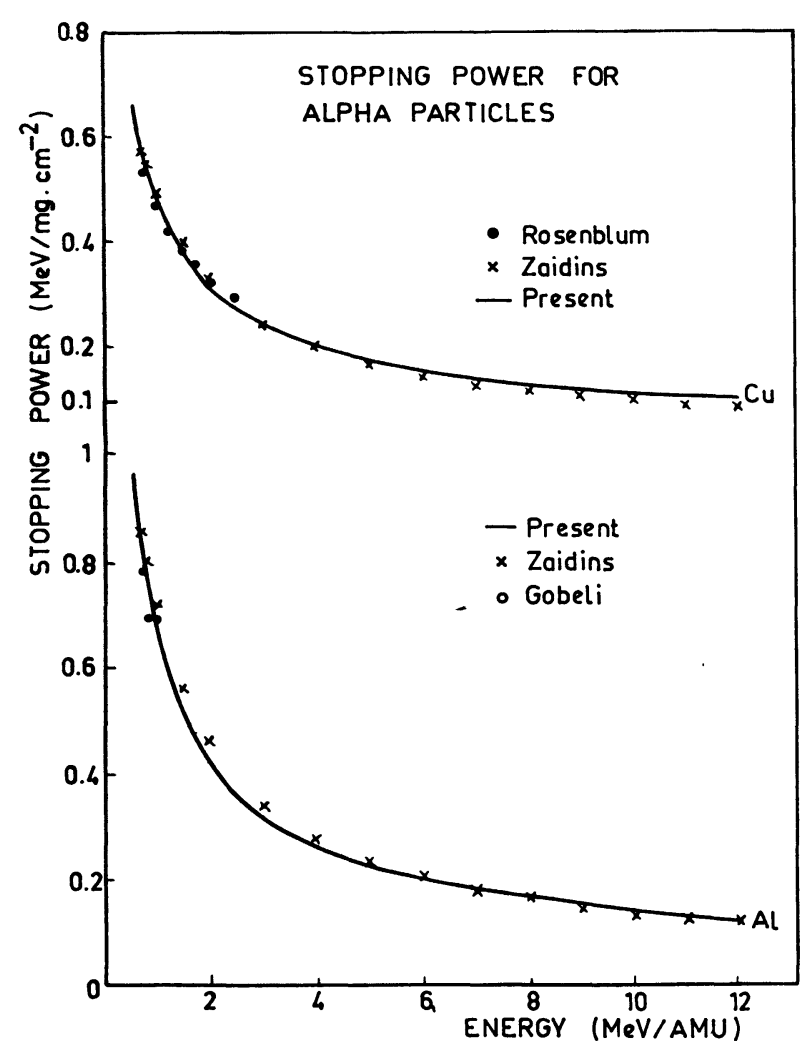

FIg. 3. - Empirical values for stopping power of alpha particles versus energy in $\mathrm{Al}$ and $\mathrm{Cu}$ along with other authors values.

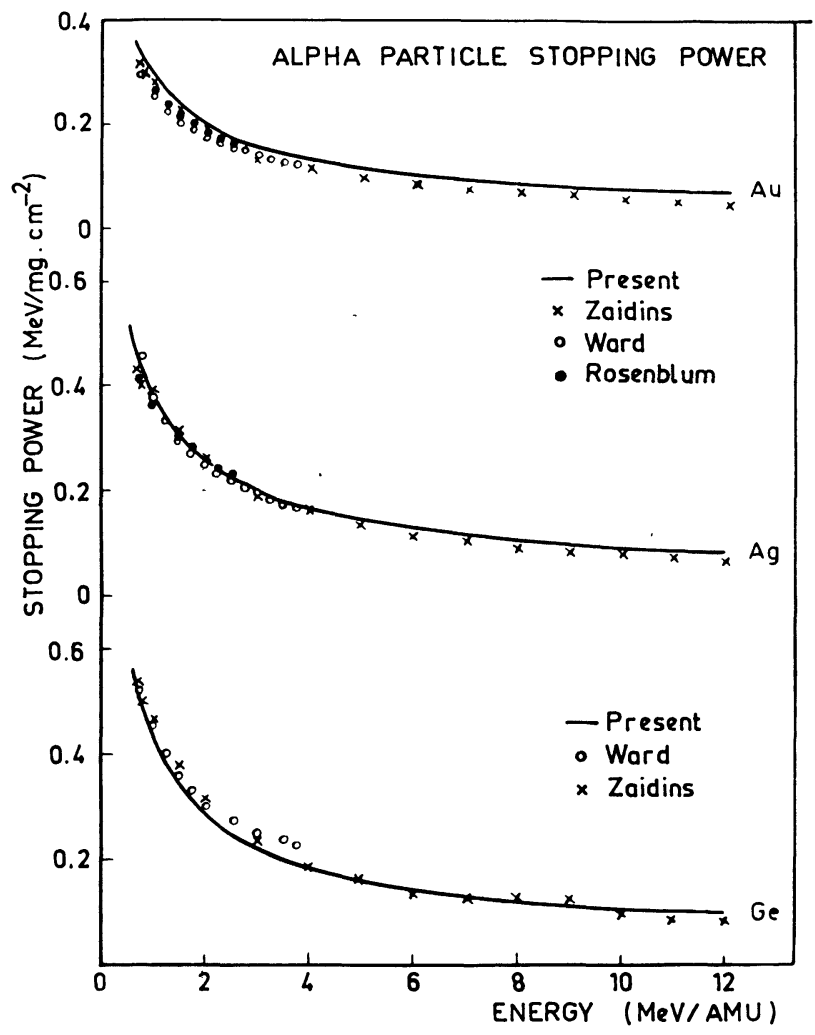

FIG. 4. - Empirical values for stopping power of alpha particles versus energy in $\mathrm{Ge}, \mathrm{Ag}$ and $\mathrm{Au}$ along with other workers values.

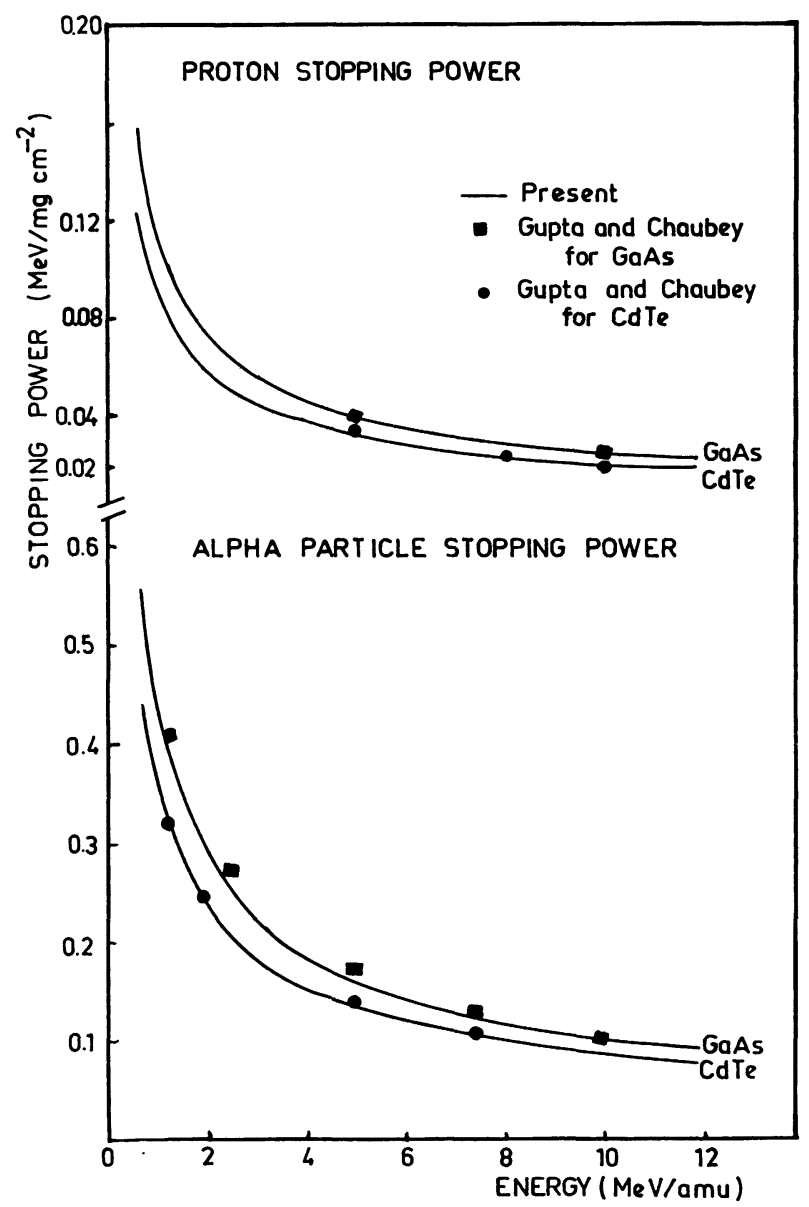

Fig. 5. - Empirical values for stopping power of protons and alpha particles versus energy in GaAs and $\mathrm{CdTe}$ along with our earlier results $[33,34]$. 


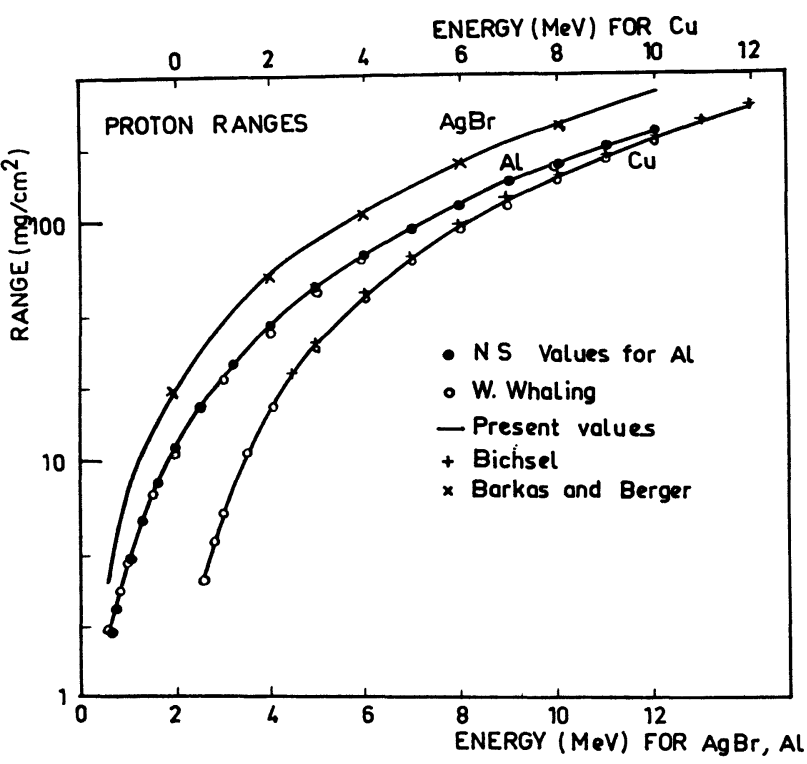

Fig. 6. - Empirical values for proton ranges versus energy in $\mathrm{Al}, \mathrm{Cu}$ and $\mathrm{AgBr}$ along with other authors results.

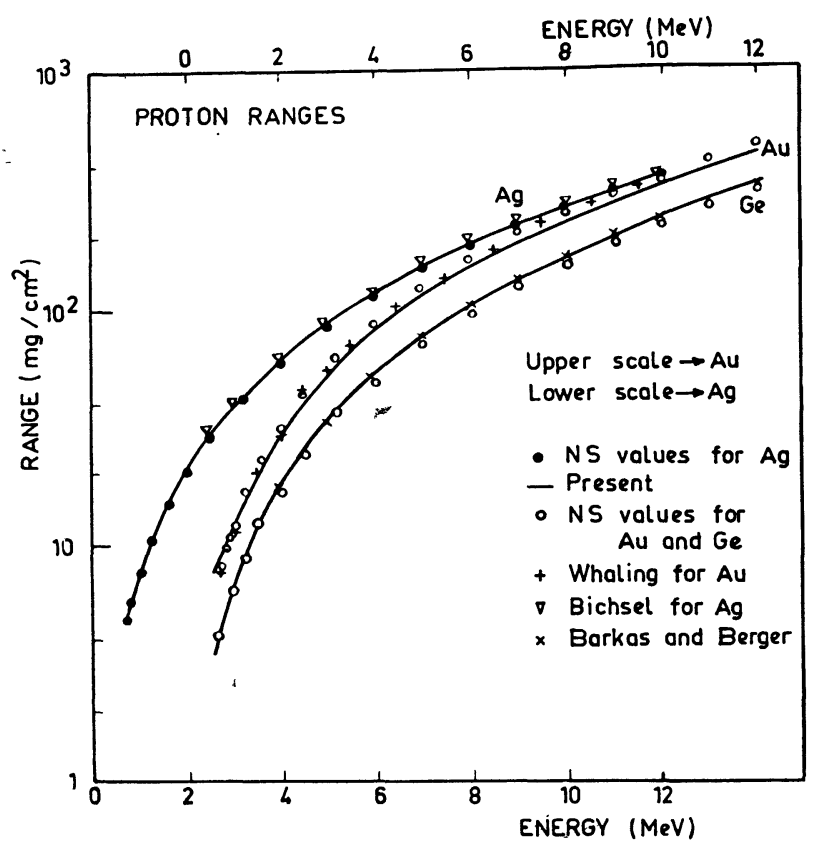

Fig. 7. - Empirical results for proton ranges versus energy in $\mathrm{Ge}, \mathrm{Ag}$ and $\mathrm{Au}$ along with other workers values.

and CdTe of protons and alpha particles. To check the validity of the present relations for compound materials we calculated stopping power and range of protons in $\mathrm{AgBr}$ and compared with Barkas and Berger [5] values. The results are shown in figures 2 and 6 . We have not presented stopping power and range values of alpha particles in $\mathrm{AgBr}$ because Barkas and Berger paper does not contain results of alpha particles, therefore comparision was not possible. In table II we have given the values of constants $G_{\mathrm{p}}$ and $G_{\alpha}$ and also correction terms $E_{\mathrm{p}}, F_{\alpha}$ and $F_{\alpha \alpha}$.

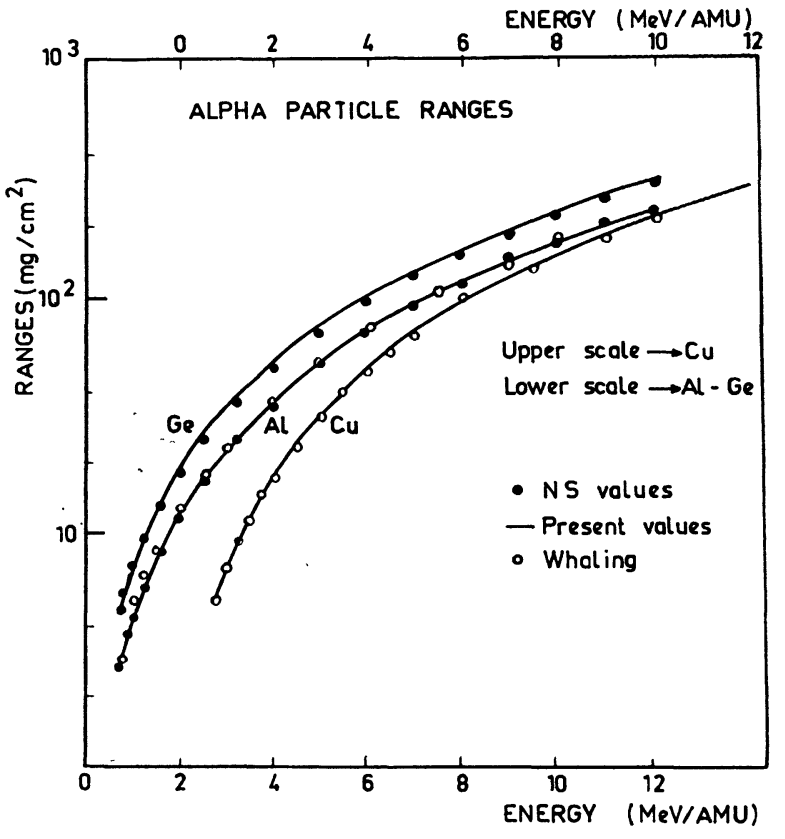

Fig. 8. - Empirical results for alpha particle ranges versus energy in $\mathrm{Al}, \mathrm{Cu}$ and $\mathrm{Ge}$ along with other workers results.

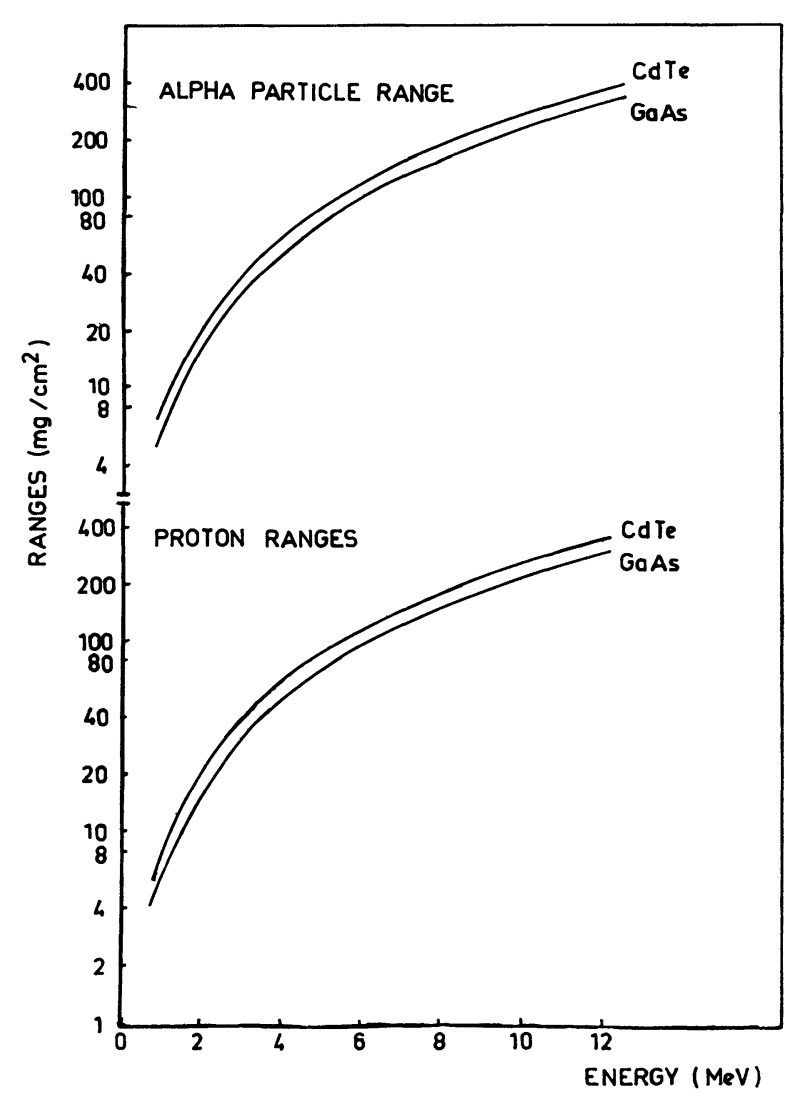

FIG. 9. - Empirical results for proton and alpha particle versus energy in $\mathrm{GaAs}$ and CdTe.

The values of stopping powers and ranges for deuteron or triton can be obtained from the tabulated values for protons by simply knowing the corresponding energy $\mathrm{E}(\mathrm{MeV})$ and multiplying by the mass 


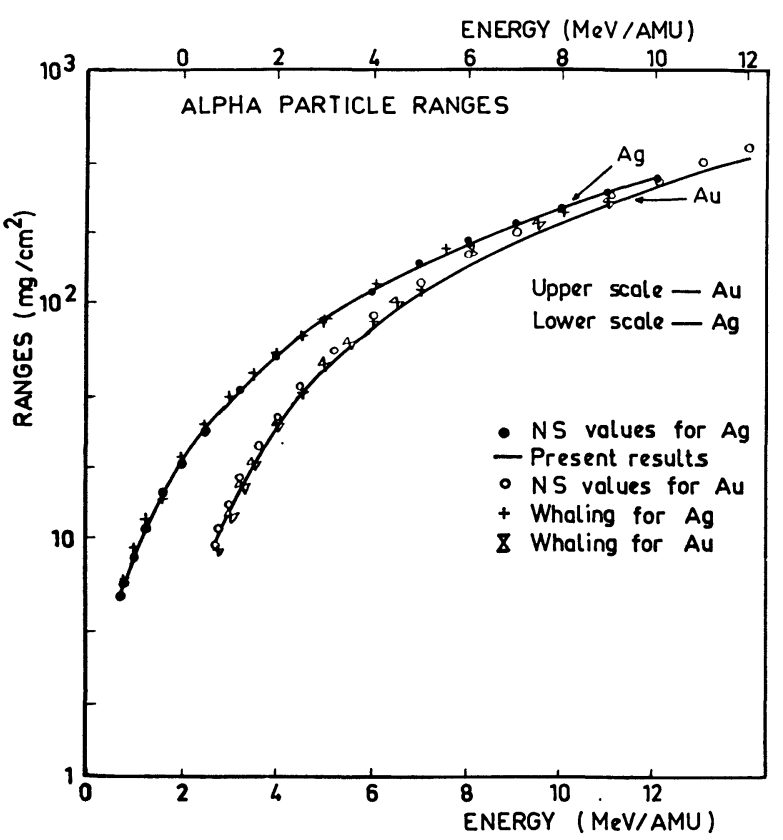

FIG. 10. - Empirical results for alpha particle ranges versus energy in $\mathrm{Ag}$ and $\mathrm{Au}$ along with other workers results.

\section{TABLE II}

$\begin{array}{cccccc}\text { Element } & G_{\mathrm{p}} & G_{\alpha} & F_{\mathrm{p}} & F_{\alpha} & F_{\alpha \alpha} \\ \overline{\mathrm{A} 1} & \overline{-} & \overline{-} & \overline{-} & \overline{-} & \overline{-} \\ \mathrm{Cu} & 4.999 & 0.882 & 0.544 & 0.818 & 0.923 \\ \mathrm{Ga} & 5.269 & 1.357 & 1.121 & 1.899 & 2.048 \\ \mathrm{Ge} & 5.384 & 1.387 & 1.149 & 1.682 & 1.840 \\ \mathrm{As} & 5.455 & 1.405 & 1.188 & 1.770 & 1.854 \\ \mathrm{Ag} & 6.359 & 1.638 & 1.338 & 1.939 & 2.132 \\ \mathrm{Cd} & 6.546 & 1.687 & 1.780 & 2.628 & 2.829 \\ \mathrm{Te} & 7.086 & 1.825 & 2.017 & 2.719 & 2.932 \\ \mathrm{Au} & 8.528 & 2.197 & 4.074 & 4.696 & 4.956\end{array}$

ratio $m^{\prime} / m_{\mathrm{p}} \simeq A^{\prime} \mid A_{\mathrm{p}}$, where $m_{\mathrm{p}}$ is the mass of proton and $m^{\prime}$ is the mass of deuteron or triton. $A^{\prime}$ and $A_{\mathrm{p}}$ pertain to the mass number of deuteron or triton and proton respectively. Similarly ranges and stopping powers of alpha particles $\left(\mathrm{He}^{4}\right)$ can be converted into the ranges and stopping powers of $\mathrm{He}^{3}$.

6. Comparision with other existing results. 6.1 Proton STOPPING POWER. - The percentage difference (D) between the stopping power calculated from eq. (1) and the corresponding values obtained from NS tables are shown in figure 11 for $\mathrm{Al}, \mathrm{Ge}, \mathrm{Zr}$, $\mathrm{Ag}, \mathrm{Eu}$ and $\mathrm{Au}$. It is evident from the figure that the empirical values for $\mathrm{Al}, \mathrm{Zr}$ and $\mathrm{Ag}$ are in good agreement with NS values within an accuracy of $5 \%$ from 0.7 to $12 \mathrm{MeV}$. The empirical values for $\mathrm{Ge}$ from 1.5 to $7 \mathrm{MeV}$ lie below the NS values by a maximum difference of $6.5 \%$. The Barkas and Berger [5] proton stopping powers for $I=340 \mathrm{eV}$, which is nearly equal to Ge ionization potential $(I=343 \mathrm{eV})$,

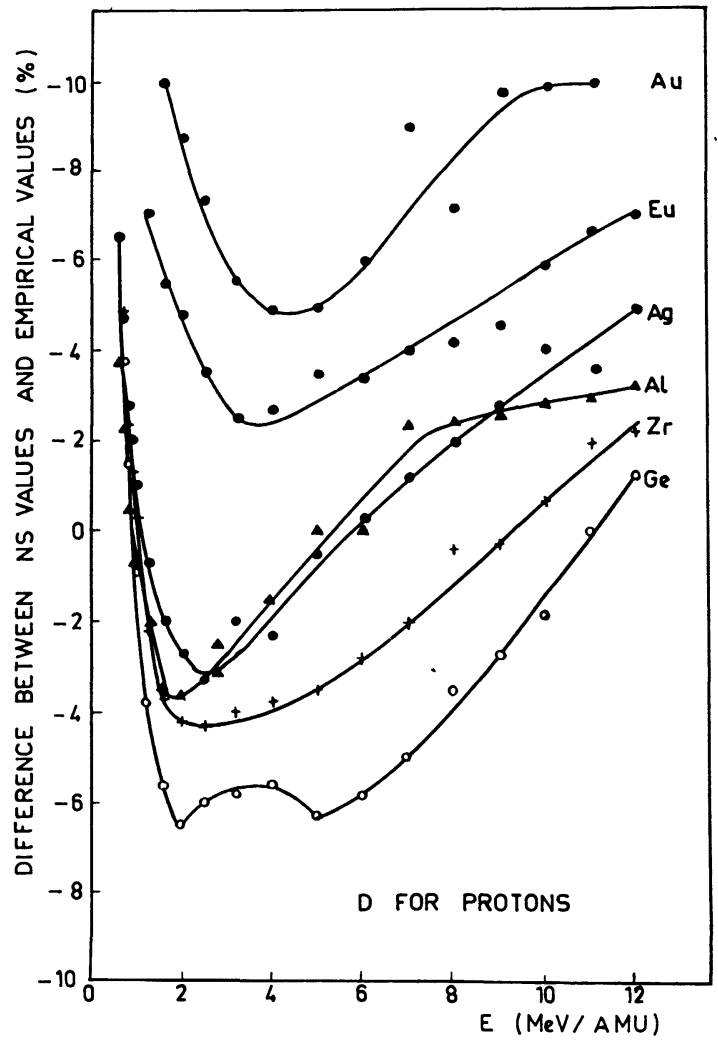

FIG. 11. - Percentage difference $(D)$ between NS and our empirical values of the stopping powers versus energy of protons for $\mathrm{Al}, \mathrm{Ge}, \mathrm{Zr}, \mathrm{Ag}, \mathrm{Eu}$ and $\mathrm{Au}$.

are also lower than NS values and the maximum difference is $7 \%$ at $6 \mathrm{MeV}$. But our values for Ge agree with that of Barkas and Berger, the maximum deviation occuring between 2 to $4 \mathrm{MeV}$. In this region the deviation is $5 \%$. Above it reduces to $2 \%$. For $\mathrm{Eu}$ and $\mathrm{Au}$ our values are higher than NS values and do not agree well on the ends of the curve (Fig. 11).

From the curves shown in these figures, one also infers that the percentage difference shows a systematic behaviour with energy as well as with atomic number. It can be noticed from figures 1 and 2 that our results for proton stopping power for $\mathrm{Al}, \mathrm{Cu}$ and $\mathrm{Ag}$ are also in excellent agreement with Bichsel [7], Anderson et al. [29] and Barkas and Berger [5] from 0.7 to $12 \mathrm{MeV}$. But for $\mathrm{Au}$ in general the agreement below $2.5 \mathrm{MeV}$ is rather poor and above this value the agreement is within $6 \%$. The comparision between our results and results of Green et al. [32] for germanium is within $3 \%$ from $0.7 \mathrm{MeV}$ to $1.0 \mathrm{MeV}$. Our empirical values of stopping power of $\mathrm{AgBr}$ are in good agreement with those of Barkas and Berger [5]. The present results for stopping power of proton in $\mathrm{CdTe}$ and GaAs as shown in figure 5 are within $5 \%$ to our earlier theoretical results [33], [34] :

We have also shown in the figures 1,2 and 3 the stopping powers calculated from Zaidins [27] empirical formula. It can be easily seen from the curves that our empirical values are closer to both experimental 
and theoretical results than the values calculated by Zaidins.

6.2 AlPha Particle STOPPING POWer. - In figure 12 we have shown the percentage difference

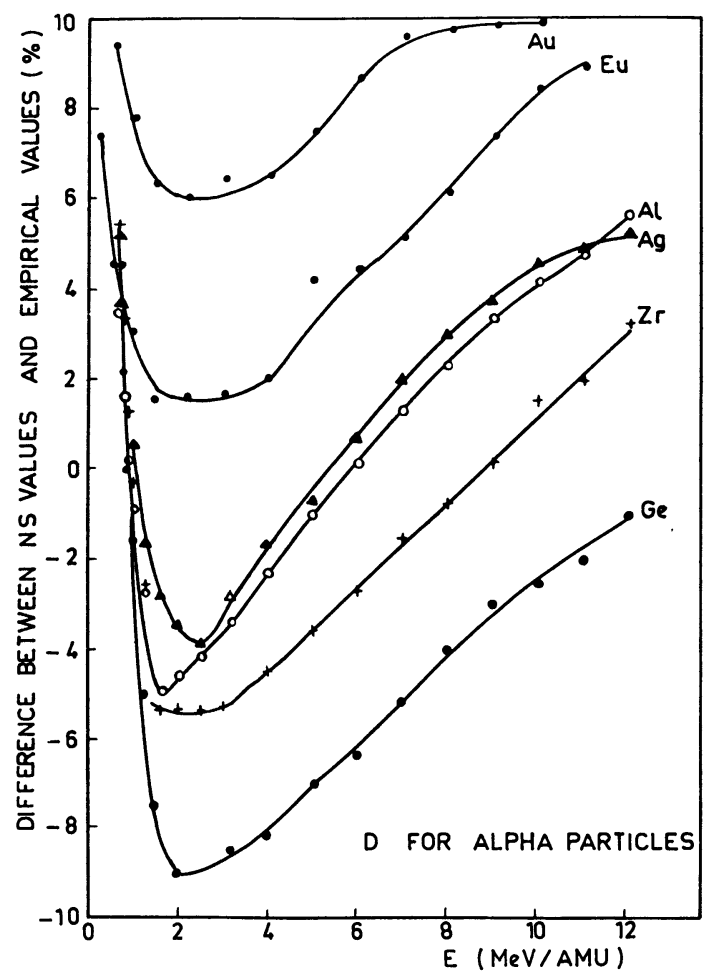

FIG. 12. - Percentage differences $(D)$ between NS values and our empirical values of the stopping powers versus energy of alpha particles for $\mathrm{Al}, \mathrm{Ge}, \mathrm{Zr}, \mathrm{Ag}, \mathrm{Eu}$ and $\mathrm{Au}$.

(D) between the empirical values calculated using eq. (5) and those obtained from NS tables. It can be inferred from these figures that NS values for $\mathrm{Al}, \mathrm{Zr}$ and $\mathrm{Ag}$ seem to be in good agreement, i.e. within $5.5 \%$, with our results. However, in the case of germanium from 1.25 to $7 \mathrm{MeV} / \mathrm{amu}$ the errors are between 5 to $9 \%$. For $\mathrm{Eu}$ and $\mathrm{Au}$, the $D$ is very large below $1.5 \mathrm{MeV} / \mathrm{amu}$ and above is the agreement is within $10 \%$. Below $4 \mathrm{MeV}$ empirical values are systematically higher than the NS values by about $5.5 \%$. In the energy range 3 to $15 \mathrm{MeV}$ Ward et al. [35] have shown that their experimental values are larger than NS values by $5 \%$ below $4 \mathrm{MeV}$. On the contrary above $4 \mathrm{MeV}$ the order is reversed. Our empirical values also compare similarly to the NS results. It can be seen in figures 3 and 4 that the Rosenblum values [36] are in excellent agreement with out values for $\mathrm{Cu}$ and $\mathrm{Ag}$ while for $\mathrm{Au}$ our values are higher than Rosenblum's. Gobeli's [18] aluminium values between 3 and $4 \mathrm{MeV}$ agree well with our empirical values. In the case of Ag, the values of Ward et al., Rosenblum, Zaidins and our results are in good agreement. The present results for stopping power of alpha particles in GaAs and CdTe as shown in figure 5 are within $5 \%$ to our earlier results [33, 34].
6.3 Proton RANGes. - The proton ranges calculated from eq. (14) are plotted against energy in figures 6,7 , and 9 . For $\mathrm{Al}$, the ranges calculated by us are very close to the ranges given by NS and Whaling. Since NS have not tabulated data for $\mathrm{Cu}$, we have compared the values with those of Bichsel and Whaling. Bichsel's $\mathrm{Cu}$ values are higher by 1 to $3 \%$ than Whaling's. Our empirical results also coincide well with Bichsel and NS data. Comparision of proton ranges in germanium with NS reveals that the maximum disagreement in the middle of the present energy range is about $6 \%$, where empirical results are higher than NS values. For Ge, we could not get other suitable experimental data for critical comparision. But the Barkas and Berger proton ranges are higher than NS values. When we compared our empirical values to Barkas and Berger we found that the worst error is $4 \%$ only. The $\mathrm{AgBr}$ empirical values more or less coincide with the Barkas and Berger values. But our $\mathrm{Au}$ results are higher than the other existing results as shown in figure 7 . We can conclude that the values presented in figure 9 for GaAs and CdTe for proton ranges are expected to be good enough for experimental purposes.

6.4 Alpha Particle Ranges. - The range of alpha particles calculated from eq. (17) and (20) are presented in figures 8,9 and 10 with the NS and Whaling results. It can be inferred that the results for $\mathrm{Al}, \mathrm{Cu}$ and $\mathrm{Ag}$ are in good agreement to the NS as well as with the Whaling data. The agreement is $3 \%$. For Ge again in the middle energy range the maximum deviation is seen with NS values and its order is about $6 \%$. In the case of gold the present values are lower than the Whaling and NS results as expected. In fact Whaling's values are systematically lower than NS values. Up to about $4 \mathrm{MeV} / \mathrm{amu}$ empirical results agree within $5 \%$ with Whaling's values and above it the error increases. The alpha particle ranges shown in figure 9 for GaAs and CdTe could not be compared as other results are not available.

7. Practical applications of empirical relations. Easy empirical formulas are very helpful in the study of energy straggling, target foil thickness estimation and in particle identification studies. The important aspect of a such problem is to know the energy loss incurred by the charged particle during the course of their passage in the absorber. The energy loss in any absorber of uniform thickness can be calculated by

$$
R(E)=R\left(E_{\mathrm{R}}\right)+T
$$

where $R(E)$ is the range at initial energy $E$ and $R\left(E_{\mathrm{R}}\right)$ corresponds to the range at residual energy $E_{\mathrm{R}}$ after it has passed thickness $T$. Generally, $E_{\mathrm{R}}$ is obtained by interpolation from the tables. The table should show the regular spacing for interpolation purposes.

The remaining energy $E_{\mathrm{R}}$ can be calculated using relations ((11), (14) and (17)) as follows. Let us first 
calculate the proton energy loss in thickness $T(\mathrm{mg} /$ $\mathrm{cm}^{2}$ ). Substituting eq. (11) into eq. (22), we get (let $\left.m_{\mathrm{p}}=1\right)$

$$
\begin{aligned}
G_{\mathrm{p}} E^{1.85} Z^{-0.145 \log E} & +F_{\mathrm{p}}= \\
= & G_{\mathrm{p}} E_{\mathrm{R}}^{1.85} Z^{-0.145 \log E_{\mathrm{R}}}+F_{\mathrm{p}}+T .
\end{aligned}
$$

We take $F_{\mathrm{p}}$ as a correction term and hence it cancels out. Therefore,

$E_{\mathrm{R}}^{1.85} Z^{-0.145 \log E_{\mathrm{R}}}=\left[\frac{G_{\mathrm{p}} E^{1.85} Z^{-0.145 \log E}-T}{G_{\mathrm{p}}}\right]$.

Taking the logarithm of both sides we get,

$$
\begin{aligned}
\log E_{\mathrm{R}}[1.85- & 0.145 \log Z]= \\
= & \log \left[\frac{G_{\mathrm{p}} E^{1.85} Z^{-0.145 \log E}-T}{G_{\mathrm{p}}}\right] .
\end{aligned}
$$

It is very easy to handle eq. (24) to calculate the residual energy. Similar equations can be written for alpha particles from eq. (14) and (17). The factor $(1.85-0.145 \log Z)=K_{\mathrm{ZP}}$ is a constant for a particular medium for atomic number $Z$. eq. (24) reduces to

$E_{\mathrm{R}}=\operatorname{Anti} \log \left[\log \left\{\frac{G_{\mathrm{p}} E^{1.85} Z^{-0.145 \log E}-T}{G_{\mathrm{p}}}\right\} / k_{\mathrm{ZP}}\right]$.

The energy loss is equal to $\left(E-E_{\mathrm{R}}\right)$. Provided we accept a little error, we can write for alpha particles the residual energy using eq. (14) as,

$E_{\alpha \mathrm{R}}=$ Anti $\log \times$

$$
\left[\log \left\{\frac{m_{\alpha} G_{\alpha} E^{1.84} Z^{-0.145 \log E}-T}{m_{\alpha} G_{\alpha}}\right\} / k_{z \alpha}\right]
$$

where

$$
K_{z \alpha}=[1.84-0.145 \log Z] .
$$

The units of $E$ used in the above expressions are $\mathrm{MeV} /$ amu.

In many nuclear physics problems, one requires the accurate measurement of target thickness. A fairly accurate determination of absorber thickness is done by exposing them to a spectrum of alpha particles with ranges of the order of absorber thickness. The measured energy loss in conjunction with known stopping power data at that energy is used to determine the thickness. With the help of multichannel analyser and detector one can measure the residual energy and then thickness of the absorber can be calculated from eq. (26). Sykes and Harris [37] during the study of energy straggling of alpha particles in thick absorbers have used the same technique to obtain aluminium absorber thickness. In figure 13 we have shown resi-

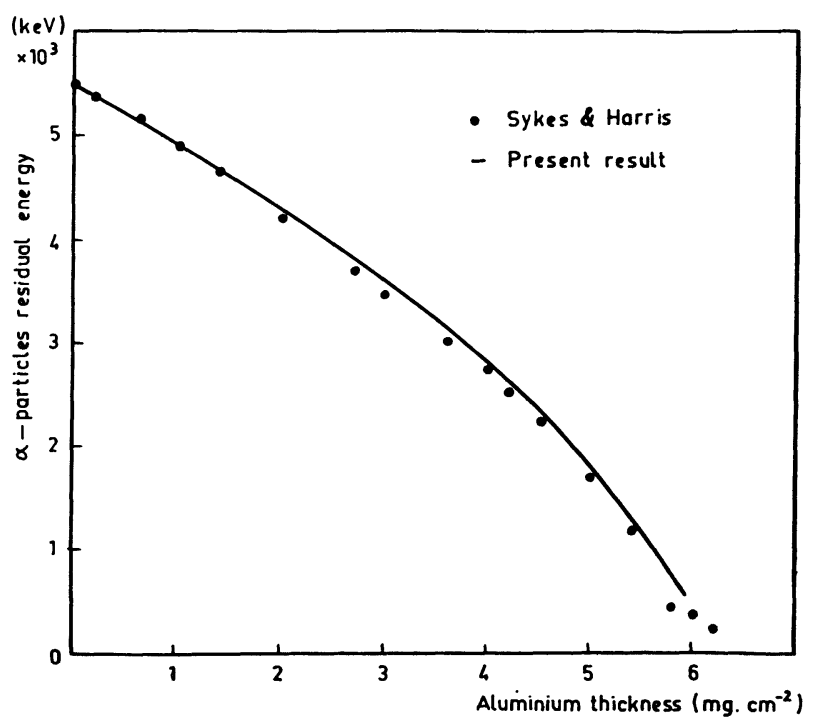

Fig. 13. - Residual energy of alpha particles as a function of $\mathrm{Al}$ absorber thickness, comparision of our results with Sykes and Harris is also shown.

dual energy calculated from eq. (26) as a function of aluminium absorber thickness. They are also compared with Sykes and Harris [37] results. We found that up to $1.4 \mathrm{mg} / \mathrm{cm}^{2}$ both the thickness more or less coincide and above it our values are a little higher than their values.

\section{References}

[1] TAylor, A. E., Rep. Prog. Phys. 15 (1952) 49.

[2] Bethe, H. A. and Askin, J., Experimental Nuclear Physics. Pt. 2 vol. 1, edited by E. Segre (John Wiley and Sons), 1952.

[3] Allision, S. K. and Warshaw, S. D., Rev. Mod. Phys. 25 (1953) 779.

[4] Uehling, E. A., Ann. Rev. Nucl. Sci. 4 (1954) 315.

[5] Barkas, W. H. and Berger, M. J., NAS-NCR Publication No. 1133, p. 103.

[6] Whaling, W., Hand. Phys. 34 (1958) 193.

[7] BiChSEL, H., American Institute of Physics, Handbook 80 (1963).

[8] GeIGER, H., Proc. R. Soc. A 83 (1910) 492.

[9] Briggs, G. H., Proc. R. Soc. A 139 (1933) 638.

[10] Rutherford, E., Williams, C. E. W. and Lewis BowDEN, B. V., Proc. R. Soc. A 139 (1933) 617.
[11] Blackett, P. M. S. and Lees, D. S., Proc. R. Soc. A 134 (1932) 658.

[12] Rogers, F. T. and Rogers, M. M., Phys. Rev. 53 (1938) 713.

[13] Meyer, F., Akad, Wiss Wien Ber. 144 (1935) 317.

[14] Rogozinski, A., J. Physique Rad. 12 (1951) 955.

[15] Wilson, R. R., Phys. Rev. 71 (1947) 385.

[16] Cook, C. J., Jones, E. and Grovensen, T., Phys. Rev. 91 (1953) 1417.

[17] Heller, Z. H. and Tendam, D. J., Phys. Rev. 84 (1951) 905.

[18] Gobeli, G. W., Phys. Rev. 103 (1956) 275.

[19] Sternheimer, R. M., Phys. Rev. 115 (1959) 137.

[20] Bichsel, H., Mozley, R. F. and Aron, W. A., Phys. Rev. 105 (1957) 1788.

[21] Barouch, E., Nucl. Instrum. Meth. 61 (1968) 113.

[22] SKYrme, D. J., Nucl. Instrum. Meth. 57 (1967) 61. 
[23] Turos, A., Nucl. Instrum. Meth. 64 (1968) 208.

[24] BiCHSEl, H. and TACHAlaev, C., UCRL-17663.

[25] Pierce, T. E. and Blann, M., Phys. Rev. 173 (1968) 390.

[26] ChU, W. K. and Powers, D., Phys. Rev. 187 (1969) 478.

[27] Zaidins, C., Nucl. Instrum. Meth. 120 (1974) 125.

[28] NorthCliffe, L. C. and Schilling, R. F., Nuclear Data A 7 (1970) 233.

[29] Anderson, H. H., Hanke, C. C., Sorensen, H. and VAJdA, P., Phys. Rev. 153 (1967) 338.

[30] Booth, W. and Grant, I. S., Nucl. Phys. 63 (1965) 481

[31] BARKAS, W. H., Nuclear Research Emulsions (Academic Press, New York), 1963.
[32] Green, D. W., Cooper, J. N. and Harris, J., Phys. Rev. 98 (1955) 466.

[33] Gupta, H. V. and ChaUbey, A. K., Nucl. Instrum. Meth. 108 (1973) 339.

[34] Gupta, H. V. and ChaubeY, A. K., Nucl. Instrum. Meth. 119 (1974) 573.

[35] Ward, D., Graham, R. L. and Geiger, J. S., Cand. J. Phys. 50 (1972) 2302.

[36] Rosenblum, S., Annls. de Phys. 10 (1928) 408.

[37] Sykes, D. A. and HARRIS, S. J., Nucl. Instrum. Meth. 94 (1971) 39. 